\title{
Polymer-graft-mediated interactions between colloidal spheres
}

\author{
Jeanette Ulama, ${ }^{\dagger}$ Malin Zackrisson Oskolkova, ${ }^{\ddagger}$ and Johan Bergenholtz ${ }^{*, \dagger, \ddagger}$ \\ Department of Chemistry and Molecular Biology, University of Gothenburg, SE-41296 \\ Göteborg, Sweden, and Division of Physical Chemistry, Center of Chemistry and Chemical \\ Engineering, Lund University, SE-22100 Lund, Sweden \\ E-mail: jbergen@chem.gu.se
}

\section{Treatment of size polydis- persity}

The particles in this study are only slightly polydisperse. Nevertheless, accounting for polydispersity is essential for reliably extracting information from small-angle scattering data. For dilute systems the scattering intensity $I(q)$ is given by the so-called form factor

$$
I(q)=n\left\langle F(q)^{2}\right\rangle=n \int_{0}^{\infty} F(q)^{2} f(R) d R
$$

where $n$ is the number density, and the form amplitude $F(q)=4 \pi \Delta \varrho(\sin q R-q R \cos q R) / q^{3}$ in this case is taken as that for a homogeneous sphere with radius $R$ and excess electron density $\Delta \varrho$. We model the size distribution as a sum of two Gaussian distributions $f(R)=X_{1} f_{1}\left(R ; \bar{R}_{1}, \sigma_{1}\right)+X_{2} f_{2}\left(R ; \bar{R}_{2}, \sigma_{2}\right)$, centered on $\bar{R}_{1}$ and $\bar{R}_{2}$, respectively, with standard deviations $\sigma_{1}$ and $\sigma_{2}$. With these Gaussian distributions each normalized to unity the mole fractions of the two populations satisfy $X_{1}+X_{2}=1$. For sufficiently narrow size distributions the lower integration limit in Eq. 1 can be extended to $-\infty$ and the integral can be

\footnotetext{
*To whom correspondence should be addressed

$\dagger$ University of Gothenburg

$\ddagger$ Lund University
}

completed analytically to yield

$$
\begin{aligned}
& \left\langle F(q)^{2}\right\rangle= \\
& \left(\frac{4 \pi \Delta \varrho}{q^{3}}\right)^{2}\left(X_{1} G\left(q \bar{R}_{1}, q \sigma_{1}\right)+X_{2} G\left(q \bar{R}_{2}, q \sigma_{2}\right)\right)(2)
\end{aligned}
$$

where

$$
\begin{aligned}
& G(x, y)= \\
& \frac{1}{2}\left\{1+x^{2}+y^{2}-2 x\left(1+2 y^{2}\right) e^{-2 y^{2}} \sin (2 x)\right. \\
& \left.\quad+\left(x^{2}-3 y^{2}-4 y^{4}-1\right) e^{-2 y^{2}} \cos (2 x)\right\}
\end{aligned}
$$

with $x=q \bar{R}$ and $y=q \sigma$.

For dilute systems this representation of the system in terms of a continuous distribution is possible. However, to enable analysis of scattering curves for more concentrated systems, it is necessary to employ a discrete-mixture representation for which an integral equation theory can be solved for the partial structure factors. To specify the histogram of discrete sizes the procedure devised by D'Aguanno and Klein ${ }^{1}$ is employed. In this method the composition and particle sizes of the mixture are chosen such that the moments of the histogram match the corresponding moments of the continuous distribution (for $0 \leq n \leq 2 N-1$ )

$$
\sum_{i=1}^{N} x_{i} r_{i}^{n}=\int_{0}^{\infty} f(r) r^{n} d r
$$

Here, $x_{i}$ and $r_{i}$ and the mole fractions and radii 
of the $N$ components of the histogram distribution, and $f(r)$ is the continuous distribution of radii normalized such that $\int_{0}^{\infty} f(r) d r=1$.

In a parallel study ${ }^{2}$ of the form factor it was found that the scattering can be quantitatively reproduced by a slightly distorted Gaussian distribution characterized by the $\bar{R}_{1}, \sigma_{1}, \bar{R}_{2}, \sigma_{2}$, and $X_{1}$ parameter values listed in Table 1 . This Gaussian distribution and a 3-component histogram representation are shown in Fig. 1. The matching of moments $n=0$ through $n=2 N-1$ has been used to determine the mole fractions and radii. As seen in Fig. 1, this results in a form factor model that is indistinguishable from the one generated from the continuous distribution for all but the highest $q$ values. The differences that appear at higher $q$ can be removed by successively adding more components to the histogram.

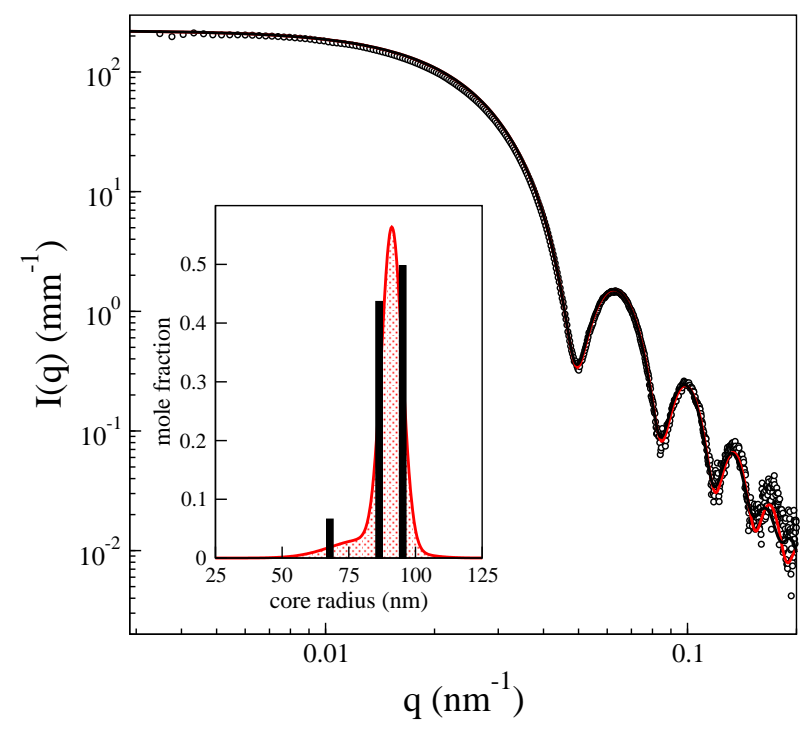

Figure 1: Form factor determined using USAXS under dilute conditions compared with form factor models resulting from a slightly distorted Gaussian size distribution and a discrete 3-component mixture. Both size distributions are shown in the inset. The radii and mole fractions of the mixture have been determined so as to match the first 6 moments of the continuous distribution following the procedure of D'Aguanno and Klein. ${ }^{1}$

In Table 1 we have also included the values of the parameters that govern the interactions needed to model the non-dilute SAXS data in
Figs. 3 and 4.

\section{References}

(1) D'Aguanno, B.; Klein, R. IntegralEquation Theory of Polydisperse Yukawa Systems. Phys. Rev. A 1992, 46, 7652.

(2) Ulama, J.; Jonsson, K.; Oskolkova, M. Z.; Bergenholtz, J. to be published. 
Table 1: Parameters associated with the model fits of the SAXS data of Figs. 3 and 4.

\begin{tabular}{ll|llllll}
\hline$\left[\mathrm{Na}_{2} \mathrm{CO}_{3}\right]$ & wt. $\%$ & $\bar{R}_{1}$ & $\sigma_{1}$ & $\bar{R}_{2}$ & $\sigma_{2}$ & $X_{1}$ & $\delta$ \\
\hline 0 & 5.6 & 81.0 & 12.7 & 91.2 & 3.8 & 0.16 & 6 \\
0 & 10.5 & 81.0 & 12.7 & 91.2 & 3.8 & 0.16 & 6 \\
0 & 15.5 & 81.0 & 12.7 & 91.2 & 3.8 & 0.16 & 6 \\
0 & 20.3 & 81.0 & 12.7 & 91.2 & 3.8 & 0.16 & 6 \\
\hline$\left[\mathrm{Na}_{2} \mathrm{CO}_{3}\right]$ & wt.\% & $\bar{R}_{1}$ & $\sigma_{1}$ & $\bar{R}_{2}$ & $\sigma_{2}$ & $X_{1}$ & $B / B_{0}^{\mathrm{HS}}$ \\
\hline 0.33 & 13.0 & 81.0 & 12.7 & 91.2 & 3.8 & 0.16 & 0.68 \\
0.43 & 13.0 & 81.0 & 12.7 & 91.2 & 3.8 & 0.16 & 0.60 \\
0.54 & 13.0 & 81.0 & 12.7 & 91.2 & 3.8 & 0.16 & 0.27 \\
\hline
\end{tabular}

